Int. J. Contemp. Math. Sci., Vol. 2, 2007, no. 11, 529 - 536

\title{
A Schur-type Theorem on Indefinite Quaternionic Kähler Manifolds
}

\author{
Gabriel Eduard Vîlcu \\ Department of Mathematics and Computer Science \\ "Petroleum-Gas" University of Ploieşti \\ Bulevardul Bucureşti, Nr. 39, Ploieşti, România \\ gvilcu@mail.upg-ploiesti.ro
}

\begin{abstract}
In this paper we give a Schur-type theorem on indefinite quaternionic Kähler manifolds.
\end{abstract}

\section{Mathematics Subject Classification: 53C15}

Keywords: quaternionic Kähler manifolds, quaternionic space forms

\section{Introduction}

The study of quaternionic Kähler manifolds with indefinite metric was initiated by J.D. Perez and F.G. Santos ([10]). Afterwards, this class of manifolds have been intensively studied by many authors ([5], [7], [9], [11], [12]). In section 2 we recall the definition and basic properties of indefinite quaternionic Kähler manifolds.

In section 3 we give some curvature properties of indefinite quaternionic Kähler manifolds. In section 4 we prove a Schur-type theorem on indefinite quaternionic Kähler manifolds.

\section{Preliminaries}

Let $M$ be a differentiable manifold of dimension $n$ and assume that there is a rank 3-subbundle $\sigma$ of $\operatorname{End}(T M)$ such that a local basis $\left\{J_{1}, J_{2}, J_{3}\right\}$ exists of sections of $\sigma$ satisfying:

$$
\left\{\begin{array}{c}
J_{\alpha}^{2}=-I d, \forall \alpha=\overline{1,3} \\
J_{1} J_{2}=-J_{2} J_{1}=J_{3}
\end{array}\right.
$$


Then the bundle $\sigma$ is called a quaternionic structure on $M$ and $\left\{J_{1}, J_{2}, J_{3}\right\}$ is called canonical local basis of $\sigma$. Moreover, $(M, \sigma)$ is said to be an almost quaternionic manifold. It is easy to see that any almost quaternionic manifold is of dimension $n=4 m$.

A pseudo-Riemannian metric $g$ is said to be adapted to the quaternionic structure $\sigma$ if it satisfies:

$$
g\left(J_{\alpha} X, J_{\alpha} Y\right)=g(X, Y), \forall \alpha=\overline{1,3}
$$

for all vector fields $X, Y$ on $M$ and any local basis $\left\{J_{1}, J_{2}, J_{3}\right\}$ of $\sigma$. Moreover, $(M, \sigma, g)$ is said to be an indefinite almost quaternionic manifold. It is clear that the signature of $g$ is $s=4 u, u \geq 0$.

If the bundle $\sigma$ is parallel with respect to the Levi-Civita connection $\nabla$ of $g$, then $(M, \sigma, g)$ is said to be an indefinite quaternionic Kähler manifold. Equivalently, locally defined 1 -forms $\omega_{1}, \omega_{2}, \omega_{3}$ exist such that:

$$
\left\{\begin{array}{r}
\nabla_{X} J_{1}=\omega_{3}(X) J_{2}-\omega_{2}(X) J_{3} \\
\nabla_{X} J_{2}=-\omega_{3}(X) J_{1}+\omega_{1}(X) J_{3} \\
\nabla_{X} J_{3}=\omega_{2}(X) J_{1}-\omega_{1}(X) J_{2}
\end{array}\right.
$$

for any vector field $X$ on $M$.

We remark that any indefinite quaternionic Kähler manifold is an Einstein manifold, provided that $\operatorname{dim} M>4$ (see [5], [10]).

\section{Curvature Properties of Indefinite Quater- nionic Kähler Manifolds}

Let $(M, \sigma, g)$ be an indefinite quaternionic Kähler manifold. If $R$ is the curvature tensor of $M$, then we have:

$$
\left\{\begin{array}{r}
{\left[R(X, Y), J_{1}\right]=F_{3}(X, Y) J_{2}-F_{2}(X, Y) J_{3}} \\
{\left[R(X, Y), J_{2}\right]=-F_{3}(X, Y) J_{1}+F_{1}(X, Y) J_{3}} \\
{\left[R(X, Y), J_{3}\right]=F_{2}(X, Y) J_{1}-F_{1}(X, Y) J_{2}}
\end{array}\right.
$$

for any vector fields $X, Y$ on $M$, where:

$$
\left[R(X, Y), J_{\alpha}\right]:=R(X, Y) J_{\alpha}-J_{\alpha} R(X, Y), \forall \alpha=\overline{1,3}
$$

and

$$
F_{1}=d \omega_{1}+\omega_{2} \wedge \omega_{3}, F_{2}=d \omega_{2}+\omega_{3} \wedge \omega_{1}, F_{3}=d \omega_{3}+\omega_{1} \wedge \omega_{2} .
$$

From (4) we obtain:

$\left\{\begin{array}{l}R\left(X, Y, J_{1} Z, J_{1} W\right]=R(X, Y, Z, W)+F_{2}(X, Y) g\left(J_{2} Z, W\right)+F_{3}(X, Y) g\left(J_{3} Z, W\right) \\ R\left(X, Y, J_{2} Z, J_{2} W\right]=R(X, Y, Z, W)+F_{1}(X, Y) g\left(J_{1} Z, W\right)+F_{3}(X, Y) g\left(J_{3} Z, W\right) \\ R\left(X, Y, J_{3} Z, J_{3} W\right]=R(X, Y, Z, W)+F_{1}(X, Y) g\left(J_{1} Z, W\right)+F_{2}(X, Y) g\left(J_{2} Z, W\right)\end{array}\right.$

for any vector field $X, Y, Z, W$ on $M$. 
Proposition 3.1 Let $\left(M^{4 m}, \sigma, g\right)$ be an indefinite quaternionic Kähler manifold. If $\left\{E_{i}\right\}_{i=\overline{1,4 m}}$ is an adapted local pseudo-orthonormal reper (i.e. $E_{m+i}=$ $\left.J_{1} E_{i}, E_{2 m+i}=J_{2} E_{i}, E_{3 m+i}=J_{3} E_{i}, \forall i=\overline{1, m}\right)$, then the relation:

$$
F_{\alpha}(X, Y)=\frac{1}{2 m} \sum_{i=1}^{4 m} \epsilon_{i} R\left(X, Y, J_{\alpha} E_{i}, E_{i}\right)
$$

holds for any $\alpha=1,2,3$ and any local vector fields $X, Y$ on $M$, where $\epsilon_{i}=$ $g\left(E_{i}, E_{i}\right)$.

Proof. If we take $Z=J_{1} E_{i}$ and $W=E_{i}$ in (7), we obtain:

$$
F_{1}(X, Y)=\epsilon_{i}\left[R\left(X, Y, J_{3} E_{i}, J_{2} E_{i}\right)+R\left(X, Y, J_{1} E_{i}, E_{i}\right)\right] .
$$

Since $\left\{E_{i}\right\}_{i=\overline{1,4 m}}$ is an adapted local pseudo-orthonormal reper, we have:

$$
\sum_{i=1}^{4 m} \epsilon_{i} R\left(X, Y, J_{3} E_{i}, J_{2} E_{i}\right)=\sum_{i=1}^{4 m} \epsilon_{i} R\left(X, Y, J_{1} E_{i}, E_{i}\right) .
$$

From (9) and (10) we derive:

$$
F_{1}(X, Y)=\frac{1}{2 m} \sum_{i=1}^{4 m} \epsilon_{i} R\left(X, Y, J_{1} E_{i}, E_{i}\right)
$$

and thus we obtain the conclusion for $\alpha=1$. Similarly we find the relation (8) for $\alpha=2$ and 3 .

Proposition 3.2 Let $\left(M^{4 m}, \sigma, g\right)$ be an indefinite quaternionic Kähler manifold. If $\left\{E_{i}\right\}_{i=\overline{1,4 m}}$ is an adapted local pseudo-orthonormal reper, then:

$$
F_{\alpha}(X, Y)=-\frac{1}{m} \sum_{i=1}^{4 m} \epsilon_{i} R\left(X, E_{i}, Y, J_{\alpha} E_{i}\right)
$$

for any $\alpha=1,2,3$ and any local vector fields $X, Y$ on $M$, where $\epsilon_{i}=g\left(E_{i}, E_{i}\right)$.

Proof. Since $\left\{E_{i}\right\}_{i=1,4 m}$ is an adapted local pseudo-orthonormal reper, we remark that:

$$
\sum_{i=1}^{4 m} \epsilon_{i} R\left(X, E_{i}, J_{\alpha} E_{i}, Y\right)=-\sum_{i=1}^{4 m} \epsilon_{i} R\left(X, J_{\alpha} E_{i}, E_{i}, Y\right)
$$

Using (12) and Bianchi identity, we have:

$$
\begin{aligned}
\sum_{i=1}^{4 m} \epsilon_{i} R\left(X, E_{i}, J_{\alpha} E_{i}, Y\right)= & \frac{1}{2} \sum_{i=1}^{4 m} \epsilon_{i}\left[R\left(X, E_{i}, J_{\alpha} E_{i}, Y\right)-R\left(X, J_{\alpha} E_{i}, E_{i}, Y\right)\right] \\
& =\frac{1}{2} \sum_{i=1}^{4 m} \epsilon_{i}\left[R\left(X, E_{i}, J_{\alpha} E_{i}, Y\right)+R\left(J_{\alpha} E_{i}, X, E_{i}, Y\right)\right] \\
& =-\frac{1}{2} \sum_{i=1}^{4 m} \epsilon_{i} R\left(E_{i}, J_{\alpha} E_{i}, X, Y\right)
\end{aligned}
$$

Finally, from (8) and (13) we obtain (11). 
Corollary 3.3 Let $\left(M^{4 m}, \sigma, g\right)$ be an indefinite quaternionic Kähler manifold with $m>1$. Then:

$$
\operatorname{Ric}(X, Y)=-(m+2) F_{\alpha}\left(X, J_{\alpha} Y\right)
$$

for any $\alpha=1,2,3$ and any local vector fields $X, Y$ on $M$.

Proof. Using Proposition 3.2 and the definition of Ricci tensor in the semiRiemmanian case, we obtain:

$$
\begin{aligned}
& \operatorname{Ric}(X, Y)=-m F_{1}\left(X, J_{1} Y\right)-F_{2}\left(X, J_{2} Y\right)-F_{3}\left(X, J_{3} Y\right), \\
& \operatorname{Ric}(X, Y)=-F_{1}\left(X, J_{1} Y\right)-m F_{2}\left(X, J_{2} Y\right)-F_{3}\left(X, J_{3} Y\right)
\end{aligned}
$$

and

$$
\operatorname{Ric}(X, Y)=-F_{1}\left(X, J_{1} Y\right)-F_{2}\left(X, J_{2} Y\right)-m F_{3}\left(X, J_{3} Y\right) .
$$

Since $m \neq 1$, from (15),(16) and (17) we deduce (14).

\section{Schur's theorem for indefinite quaternionic Kähler manifolds}

Let $(M, \sigma, g)$ be an indefinite almost quaternionic manifold. If $X \in T_{p} M, p \in$ $M$, then the 4-plane $Q(X)$ spanned by $\left\{X, J_{1} X, J_{2} X, J_{3} X\right\}$ is called a quaternionic 4-plane. A 2-plane in $T_{p} M$ spanned by $\{X, Y\}$ is called half-quaternionic if $Q(X)=Q(Y)$.

Let $\pi=S p\{X, Y\}$ be a plane tangent to $M$ at a point $p \in M$. The sectional curvature $K(\pi)$ is defined by:

$$
K(\pi)=\frac{R(X, Y, X, Y)}{g(X, X) g(Y, Y)-g(X, Y)^{2}} .
$$

It is clear that this definition makes sense only for nondegenerate plane, i.e. those satisfying $g(X, X) g(Y, Y)-g(X, Y)^{2} \neq 0$

The sectional curvature for a half-quaternionic 2-plane is called quaternionic sectional curvature. An indefinite quaternionic Kähler manifold is an indefinite quaternionic space form (denoted $M(c)$ ) if its quaternionic sectional curvatures are equal to a constant $c$.

Theorem 4.1 Let $\left(M^{4 m}, \sigma, g\right)$ be an indefinite quaternionic Kähler manifold, with $m>1$. If $p$ is a point of $M$ and the sectional curvatures of any halfquaternionic 2-plane $\pi \subset T_{p} M$ are given, then the Riemann-Christoffel curvature tensor is unique determined. 
Proof. We suppose that $R$ and $R^{\prime}$ are two Riemann-Christoffel curvature tensors on $M$, such that:

$$
R(X, Y, X, Y)=R^{\prime}(X, Y, X, Y)
$$

for any pseudo-orthonormal basis $\{X, Y\}$ of $\pi \subset T_{p} M$, with $Q(X)=Q(Y)$.

If we define $S:=R-R^{\prime}$, then we have:

$$
S(X, Y, X, Y)=0
$$

for any pseudo-orthonormal basis $\{X, Y\}$ of $\pi \subset T_{p} M$, with $Q(X)=Q(Y)$.

To complete the proof, it suffices to prove that the above identity holds true for any $X, Y \in T_{p} M$.

If $F_{1}, F_{2}, F_{3}$ and $F_{1}^{\prime}, F_{2}^{\prime}, F_{3}^{\prime}$ are locally defined 2-forms corresponding to $R$ and $R^{\prime}$ (cf. 6), then we consider:

$$
F_{\alpha}^{S}:=F_{\alpha}-F_{\alpha}^{\prime}, \forall \alpha=\overline{1,3}
$$

From (14) and (21) we obtain:

$$
F_{\alpha}^{S}(X, Y)=\frac{\operatorname{Ric}_{R}\left(X, J_{\alpha} Y\right)}{m+2}-\frac{\operatorname{Ric}_{R^{\prime}}\left(X, J_{\alpha} Y\right)}{m+2}
$$

Since $M$ is Einstein, we have $R i c_{R}=\lambda_{R} g$ and $R i c_{R^{\prime}}=\lambda_{R^{\prime}} g$, and from (22) we obtain:

$$
F_{\alpha}^{S}(X, Y)=k g\left(X, J_{\alpha} Y\right)
$$

where $k=\frac{\lambda_{R}-\lambda_{R^{\prime}}}{m+2}$.

From (7), taking account of definition of $S$, we deduce:

$$
S\left(J_{1} X, J_{3} X, J_{2} X, X\right)=-S\left(X, J_{2} X, X, J_{2} X\right)-F_{2}^{S}\left(X, J_{2} X\right) g\left(J_{3} X, J_{3} X\right)
$$

for any vector fields $X, Y, Z, W$ on $M$.

We have two possible situations.

Case 1. If $X$ is a non-lightlike vector (i.e. $g(X, X) \neq 0$ ), then we can suppose that $X$ is a unitary spacelike vector (i.e. $g(X, X)=1$ ) or unitary timelike vector (i.e. $g(X, X)=-1$ ). We consider the first situation (the second situation is completely analogous). From (20), (23) and (24) we obtain:

$$
S\left(X, J_{2} X, J_{3} X, J_{1} X\right)=k g(X, X)^{2} .
$$

Similarly, we find:

$$
S\left(X, J_{3} X, J_{1} X, J_{2} X\right)=k g(X, X)^{2}
$$

and

$$
S\left(X, J_{1} X, J_{2} X, J_{3} X\right)=k g(X, X)^{2}
$$


From (25), (26) and (27), using Bianchi identity, we derive:

$$
3 k g(X, X)^{2}=0
$$

and since $X$ is a non-lightlike vector, we deduce $k=0$ and thus we have also $F_{\alpha}^{S}=0, \forall \alpha=\overline{1,3}$. Now, we obtain easy from (7) that $S(X, Y, X, Y)=0$ for any $X, Y \in T_{p} M$.

Case 2. If $X$ is a lightlike vector (i.e. $\mathrm{g}(\mathrm{X}, \mathrm{X})=0$ ), there exists a sequence $\left\{X_{n}\right\}_{n}$ of non-lightlike tangent vectors such that $X_{n} \rightarrow X$. Using above case, we obtain $S\left(X_{n}, Y, X_{n}, Y\right)=0$, for any $n$ and any $Y \in T_{p} M$. For $n \rightarrow \infty$, we obtain $S(X, Y, X, Y)=0$ for any $X, Y \in T_{p} M$.

The proof is now complete.

Let $\left(M^{4 m}, \sigma, g\right)$ be an indefinite quaternionic Kähler manifold, with $m>1$. Let $R_{0}$ be the tensor field on $M$ given by:

$$
\begin{aligned}
R(X, Y, Z, W)= & \frac{1}{4}\left\{g(X, Z) g(Y, W)-g(X, W) g(Y, Z)+\sum_{\alpha=1}^{3}\left[g\left(X, J_{\alpha} Z\right) g\left(Y, J_{\alpha} W\right)\right.\right. \\
& \left.\left.-g\left(X, J_{\alpha} W\right) g\left(Y, J_{\alpha} Z\right)+2 g\left(X, J_{\alpha} Y\right) g\left(Z, J_{\alpha} W\right)\right]\right\}
\end{aligned}
$$

for any $X, Y, Z, W \in T_{p} M$ and any local basis $\left\{J_{1}, J_{2}, J_{3}\right\}$ of $\sigma$ at p. It is easy to see that above definition does not depend on the basis $\left\{J_{1}, J_{2}, J_{3}\right\}$ of $\sigma$ chosen on a neighborhood of any point. Moreover, $R_{0}$ satisfies the properties of Riemann-Christoffel curvature tensor:

i. $R_{0}(X, Y, Z, W)=-R_{0}(Y, X, Z, W)=-R_{0}(X, Y, W, Z)$;

ii. $R_{0}(X, Y, Z, W)=R_{0}(Z, W, X, Y)$;

iii. $R_{0}(X, Y, Z, W)+R_{0}(X, Z, W, Y)+R_{0}(X, W, Y, Z)=0$

We remark that, if $p$ is a point of $M$ and the sectional curvatures of any halfquaternionic 2-plane $\pi \in T_{p} M$ are equal to a constant $c$, then, from Theorem 4.1, we deduce that in $p$ we have: $R=c R_{0}$.

Theorem 4.2 Let $\left(M^{4 m}, \sigma, g\right)$ be an indefinite quaternionic Kähler manifold, with $m>1$. If $M$ is connected and for each $p \in M$, the sectional curvature is constant on the half-quaternionic 2-planes in $T_{p} M$, then $M$ is an indefinite quaternionic space form, having the quaternionic sectional curvature $c=\frac{\lambda}{m+2}$, where $\lambda$ is the proportionality coefficient between Ric and $g$ (since $M$ is Einstein).

Proof. From above considerations, we deduce that:

$$
R^{\prime}=R-c R_{0}=0
$$

Similarly as in Theorem 4.1, we obtain: 


$$
F_{\alpha}^{R^{\prime}}(X, Y)=k g\left(X, J_{\alpha} Y\right)
$$

where $k=\frac{\lambda}{m+2}-c$.

Since $R^{\prime}=0$, we have $F_{\alpha}^{R^{\prime}}=0$ and from (30) we derive $k=0$ (see proof of Theorem 4.1).

Thus we conclude that $c=\frac{\lambda}{m+2}$ and the proof is now complete.

\section{References}

[1] D.V. Alekseevsky, Riemannian spaces with exceptional holonomy groups, Funkcional. Anal. Prilozen, 2 (1968), 1 - 10.

[2] D.V. Alekseevsky, S. Marchiafava, Quaternionic structures on a manifold and subordinate structures, Ann. Mat. Purra Appl., 17 (1996), 205 - 273.

[3] A. Besse, Einstein manifolds, Berlin, Springer-Verlag, 1987.

[4] M. Berger, Sur le groupes d'holonomie des variétés a connexion affine et des variétés riemanniennes, Bull. Soc. Math. France, 83 (1955), 279 310 .

[5] E. Garcia-Rio, M. E. Vazquez-Abal, On the quaternionic sectional curvature of an indefinite quaternionic Kähler manifold, Tsukuba J. Math., 19 (1995), $273-284$.

[6] S. Ishihara, Quaternion Kählerian manifolds, J. Diff. Geometry, 9 (1974), $483-500$.

[7] T.H. Kang, Lightlike real hypersurfaces of indefinite quaternionic Kaehler manifolds, Indian J. Pure Appl. Math, 33 (2002), 1755 - 1766.

[8] B. O'Neill, Semi-Riemannian geometry with applications to relativity, Pure and Applied Mathematics, 103. New York-London etc.: Academic Press, 1983.

[9] J. D. Perez, Indefinite quaternion space forms II, Ann. Stiint. Univ. Al. I. Cuza Iasi, 32 (1986), 63 - 68.

[10] J. D. Perez, F. G. Santos, Indefinite quaternion space forms, Ann. Mat. Purra Appl., 132 (1982), 383 - 398.

[11] B. Şahin, R. Guneş, QR-lightlike submanifolds of indefinite quaternion Kähler manifolds, Indian J. Pure Appl. Math, 33 (2002), 1685 - 1706. 
[12] B. Şahin, R. Guneş, Lightlike real hypersurfaces of indefinite quaternion Kähler manifolds, Journal of Geometry, 75 (2002), 151 - 163.

\section{Received: October 5, 2006}

